\title{
Sudden Infant Death Syndrome: Abnormalities in Short Term Fluctuations in Heart Rate and Respiratory Activity
}

\author{
DAVID GORDON, RICHARD J. COHEN, DOROTHY KELLY, SOLANGE AKSELROD, AND \\ DANIEL C. SHANNON
}

With the technical assistance of Barney Kenet, F. Andrew Ubel, and Oswald Williams

\begin{abstract}
Children's Service, Pediatric Pulmonary Unit, Massachusetts General Hospital, Boston, Massachusetts 02114 [D.G., D.K., D.C.S., B.K., O.W.], Harvard-Massachusetts Institute of Technology Division of Health Sciences and Technology [R.J.C., S.A., D.C.S., F.A.U.] and Department of Physics [D.G., R.J.C., S.A.], Massachusetts Institute of Technology, Cambridge, Massachusetts 02139, and Department of Medicine, Brigham and Women's
\end{abstract} Hospital, Boston, Massachusetts 02115 [R.J.C.]

\begin{abstract}
Summary
In order to test the hypothesis that a defect in cardiorespiratory regulation contributes to death of infants from sudden infant death syndrome (SIDS), we analyzed the power spectra of heart rate and respiratory activity during 256-sec epochs of quiet sleep. Data were obtained from pneumogram recordings performed for $12 \mathrm{~h}$ at night on eight infants who subsequently died from SIDS and 22 age-matched control infants. We computed the heart rate and respiratory power spectra from a single epoch on each infant using an algorithm developed for an $\mathbf{8 0 8 5}$ microprocessor system dedicated to this investigation. There was no statistically significant difference between SIDS and controls based on mean respiratory and heart rates. Spectral analysis revealed enhancement of low frequency power in the 0.02 to $0.1 \mathrm{~Hz}$ band in the heart rate power spectrum in the SIDS group compared to control ( $p$ $<0.002$ ) and dispersion in respiratory frequency as determined by the respiratory band width $(p<0.00001)$. These data suggest that a predisposition to SIDS manifests itself in an abnormal pattern of fluctuations in heart rate and respiratory activity.
\end{abstract}

\section{Abbreviations}

SIDS, sudden infant death syndrome

ECG, electrocardiogram

EEG, electroencephalogram

GER, gastroesophageal reflux

HR, heart rate

The purpose of this investigation was to test the hypothesis suggested by studies of pathology $(8,17)$ and physiology $(2,10$, $13,16)$ that infants who die from SIDS share an abnormality in the regulation of the cardiovascular and respiratory systems. Given the fact that the prolonged arrest of the heart beat and respiratory activity will rapidly lead to death irrespective of the antecedent triggering events, we did not attempt to identify specific morphologic, biochemical, or physiologic mechanisms for defective regulation, but focussed exclusively on the final common pathway to death: cardiorespiratory instability.

The basis of understanding the dynamics of regulatory systems

Reprint requests may be addressed to Daniel C. Shannon, M.D., Children's Service, Pediatric Pulmonary Unit, Massachusetts General Hospital, Boston, MA 02114.

This work was supported by the Healthdyne Company, the McGraw Foundation, the Scholl Foundation, R. J. Reynolds Industries, National Science Foundation Grant ECS-8121571, and Office of Naval Research grant N00014-80-C-0520. R. J. C. is grateful for support from a Hartford Foundation Fellowship. in general has rested on the analysis of naturally occurring fluctuations in system variables (4). These fluctuations contain information concerning the spontaneously changing load on the system as well as the system's response. A useful first step in the quantitative analysis of the control system is the spectral analysis of the system variables. Prior work $(1,7,9,11)$ has emphasized the importance and relevance of the analysis of fluctuations in heart rate to assess autonomic functions. We present here the results of spectral analysis of 256-sec-long segments of the instantaneous heart and respiratory waveform in 22 control infants and eight infants who died of SIDS.

\section{MATERIALS AND METHODS}

Infants who died of SIDS diagnosed after an autopsy failed to identify the cause of death were evaluated because they all had at least one nocturnal recording of ECG and respiration at home. The indications for conducting the recording were near-SIDS (2), choking spells (2), and death from SIDS in one sibling (1) or two siblings (2). One of the latter also experienced a nearSIDS event. Thus, three had not experienced near-SIDS; in two of these, GER was identified on barium swallow. Although she had no choking spells, GER was also identified in a sibling with near-SIDS (patient 7, Table 1). The shortest interval between a spell and the nocturnal recording was 5 days (the first recording in patient 7). Near-SIDS was defined as sleep onset apnea associated with limpness and cyanosis or pallor responding only to vigorous stimulation or resuscitation. Identifiable causes of pathologic apnea were ruled out by complete blood count, ECG, EEG, chest $x$-ray, serum $\mathrm{Na}^{+}, \mathrm{K}^{+}, \mathrm{Cl}^{-}, \mathrm{HCO}_{3}^{-}$, and $\mathrm{Ca}^{2+}$, and blood sugar. Parents were instructed in the use of an electronic cardiorespiratory monitor (Healthdyne Inc., Marietta, GA) and the infants were discharged to be supported by the staff of the Pediatric Pulmonary Laboratory, Massachusetts General Hospital. Three infants were not monitored, two with GER and one sibling.

Control infants were healthy full term infants without perinatal complications and enrolled by their parents prior to birth after we explained our need to collect recordings of ECG and respiratory activity at home at intervals over the first year of life. All came from the same community hospital.

The biographic data on the SIDS infants are exhibited in Table 1. Although their gestational ages were similar, SIDS infants were an average $0.55 \mathrm{~kg}$ smaller than controls. Pregnancy was complicated by gestational diabetes mellitus in one; fetal bradycardia was noted just prior to delivery in one; none required any form 
Table 1. Biographic data

\begin{tabular}{ll}
\hline \multicolumn{1}{c}{ SIDS $(n=8)$} & Controls $(n=22)$ \\
\hline Male/female, 3:5 & $11: 11$ \\
Gestational age (weeks), 38-43 (mean $=40)$ & $38-42($ mean $=40)$ \\
Body weight $(\mathrm{kg}), 2.76-3.54($ mean $=3.16)$ & $3.18-4.12($ mean $=$ \\
& $3.71)$ \\
Near-SIDS, 5 (3 repeat) & \\
Siblings, 3 &
\end{tabular}

Siblings,

\begin{tabular}{|c|c|c|c|}
\hline Patient and sex & $\begin{array}{l}\text { Age at recording } \\
\text { (weeks) }\end{array}$ & $\begin{array}{l}\text { Age at death } \\
\text { (weeks) }\end{array}$ & $\begin{array}{l}\text { Indication } \\
\text { for } \\
\text { recording }\end{array}$ \\
\hline 1. $\mathrm{F}$ & 8 & 9 & Near-SIDS \\
\hline 2. $M$ & 8 & 12 & $\begin{array}{l}\text { Choking } \\
\text { spell }\end{array}$ \\
\hline \multirow[t]{2}{*}{ 3. $\mathrm{F}$} & 3 & 8 & Sibling \\
\hline & 7 & 8 & Sibling \\
\hline 4. $M$ & 10 & 13 & Near-SIDS \\
\hline 5. F & 4 & 5 & $\begin{array}{r}\text { Sibling, } \\
\text { near- } \\
\text { SIDS }\end{array}$ \\
\hline 6. $\mathrm{M}$ & 5 & 18 & $\begin{array}{l}\text { Choking } \\
\text { spell }\end{array}$ \\
\hline 7. $F$ & 1.5 & 4 & $\begin{array}{c}\text { Sibling, } \\
\text { near- } \\
\text { SIDS, } \\
\text { GER }\end{array}$ \\
\hline 7. $\mathrm{F}$ & 3 & 4 & $\begin{array}{c}\text { Sibling, } \\
\text { near- } \\
\text { SIDS, } \\
\text { GER }\end{array}$ \\
\hline 8. $\mathrm{F}$ & 18 & 24 & Near-SIDS \\
\hline
\end{tabular}

of resuscitation at the time of birth. In those with near-SIDS, the first spell occurred between 5 days and 10 weeks of age; one recording was obtained in six infants and two recordings were obtained in each of two infants. Death occurred between 1 and 13 weeks after the recordings. For the control group of infants, age at time of recording ranged from 1 to 26 weeks; thus, they are matched for the age range of SIDS infants. There were 11 males and 11 females.

Recordings were obtained by pneumogram technique (15). ECG was recorded from a pair of pregelled electrodes adhered to the chest wall; the reference lead was applied to one thigh. Respiratory activity was recorded from a pair of electrodes by impedance method using the Healthdyne cardiorespiratory monitor. These signals were recorded on high quality magnetic tape cassettes for 10 to $12 \mathrm{~h}$ at night. Analog signals were then transferred to hard copy at 60 times real time using a PMD-12 Oxford replay unit and four channel Mingograf spray-pen recorder with paper speed set at $50 \mathrm{~mm} / \mathrm{sec}$. At the time of replay, we sampled ECG signals using a peak detector, and recorded instantaneous heart rate along with ECG and respiratory waveform. Two SIDS infants (patients 4 and 6) exhibited abnormal amounts of periodic breathing by previously established criteria (6).

The power spectrum of a signal provides a measure of the energy content of that signal as a function of frequency, that is, the relative weight of oscillations at varying frequencies. Statistically, it characterizes the distribution of variations about the mean. In Figure 1, we present an instantaneous heart rate tracing obtained from a tachometer and its associated power spectrum. One peak in the spectrum (the "high" frequency peak) is centered at the respiratory frequency $(0.42 \mathrm{~Hz})$, and constitutes a direct measure of respiratory sinus arrhythmia which is parasympathetically mediated (1). The other, a lower frequency peak, lies in the 0.02 to $0.10 \mathrm{~Hz}$ band, and is thought to reflect heart rate fluctuations which we believe compensate for perturbations in blood pressure resulting from variations in vasomotor tone (vas- cular resistance) at those frequencies. This peak is increased with increasing parasympathetic and/or increasing sympathetic tone and is obliterated by muscarinic plus $\beta$-adrenergic blockade (1). This peak also increases under conditions of blockade of the renin-angiotensin system, presumably because of increased fluctuations in peripheral vasomotor resistance (1) following release from the damping effect of angiotensin. The power spectrum of the respiratory activity reveals a peak centered at the respiratory frequency, the width of which provides a direct measure of the dispersion in respiratory frequency (see Fig. 2).

From the analog recordings, we selected by eye the 256 -sec-

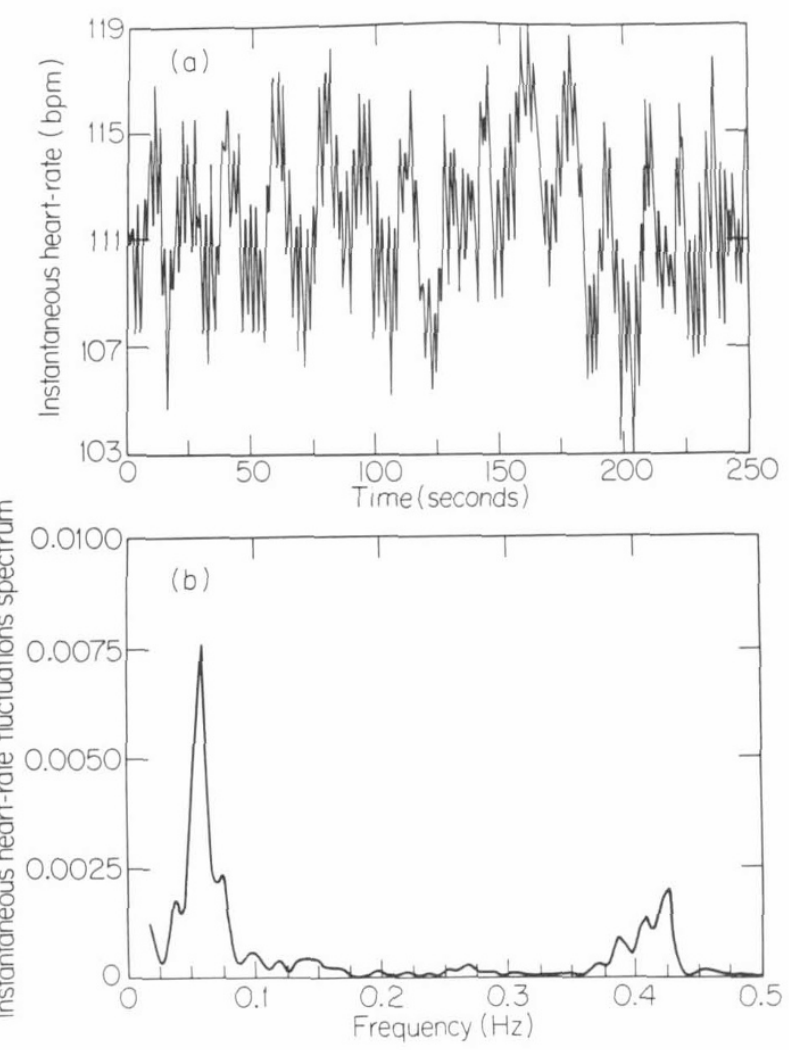

Fig. 1. Heart rate tracing and associated power spectrum.

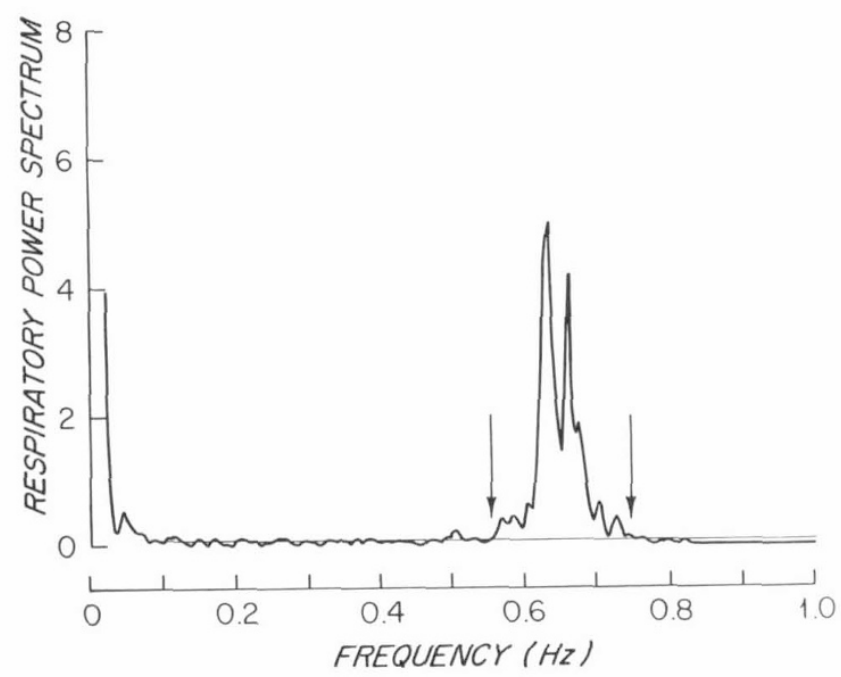

Fig. 2. Illustration of computation of respiratory band width. The solid line interpolates the background noise. The arrows indicate the band in which the respiratory peak just rises above and falls below background noise. Its width is defined as the respiratory band width. 
long segment of data from each infant during which there were the fewest fluctuations in instantaneous heart rate, the mean rate remained constant, the respiration pattern was regular without apnea or periodic breathing, and there was no evidence of a sigh or body movement, a pattern that is usually characteristic of quiet sleep (5). These least variable segments were selected independently by two observers (D. G. and D. C. S.). In order to test the reproducibility of this method of selecting these segments, we compared this visual assessment on an additional six babies to the result of the computer analysis performed independently on all available quiet sleep segments in each baby. In each case, the visual assessment of the least variable of three to eight segments per baby agreed with the computer analysis of variability; the total heart rate variance was lowest in the segment with the least variability identified visually. Clearly, by selecting the least variable 256-sec segment of data from the 10- to $12-\mathrm{h}$ record, we have rejected a vast quantity of data. However, this approach ensured that spectral analysis was performed on data corrupted the least by sigh, body movement, apnea, or state change and that the analysis was comparable for each baby.

We then identified this least variable segment of data on each tape cassette with the aid of an oscilloscope and transferred the signals to an FM, eight-channel, magnetic tape recorder (HewlettPackard 3968A). In order to compensate for fluctuations in the time base of the cassette recorder and/or replay unit, we sampled the data through a phase-locked loop amplifier that locked onto the respiratory carrier signal $(22 \mathrm{~Hz})$ used to record respiratory activity or, when available, a $60-\mathrm{Hz}$ crystal clock pulse introduced onto the cassette tape at the time of recording.

ECG data were then digitized through an 8085 microprocessor using a special peak detection algorithm developed to identify the QRS complex (12). Respiratory wave forms were sampled at $4 \mathrm{~Hz}$ after having been first passed through a $2-\mathrm{Hz}$ low pass antialiasing filter. The power spectra of the instantaneous HR and respiratory time series were calculated using an FFT algorithm. The power in the heart rate power spectrum, which is the amplitude squared, was normalized by dividing by the mean heart rate squared in order to obtain a dimensionless variable. Power in the respiratory spectrum was normalized by dividing by the total variance squared in order to determine the distribution of respiratory frequencies. For the instantaneous heart rate time series, we calculated the mean heart rate, standard deviation, as well as the integral of power within the (low) frequency band $0.02-0.10 \mathrm{~Hz}$ and the power within the (high) frequency band that coincided with the band of the principal peak in the respiratory spectrum (see Fig. 2). The latter allowed us to quantify respiratory sinus arrhythmia.

From the respiratory signal, we calculated mean respiratory frequency and the dispersion in respiratory frequency around the mean respiratory rate. We refer to this latter quantity as the respiratory band width. It is determined by measuring the width of the respiratory power spectrum at the levels at which the peak just rises above and falls below background noise (Fig. 2). Due to the subjective nature of the estimation of background noise and its importance in characterizing the respiratory band width, this width was determined independently by three different investigators (D. G., D. C. S., and B. K.). There was less than $5 \%$ interobserver variability. Because our normalization process yields the same total power in the spectrum of each baby, a change in band width reflects a change in variance of respiratory frequency around the mean value.

In order to determine the reproducibility of the analysis, we selected two 256-sec epochs of data with 120-sec overlap between the two. We then performed the analysis on each epoch separately in three babies. The mean heart rate varied by less than $0.2 \%$ and the power at low frequency by $0.3 \%$.

We determined the significance of differences in mean group values using the two-tailed Wilcoxon-Mann-Whitney rank sum test. $p$ values less than 0.05 were considered to be statistically significant.

\section{RESULTS}

There were no differences in mean values for heart rate and respiratory rate between the SIDS and control group (Tables 2 and 3; Figs. 3 and 4).

There was no difference in the magnitude of respiratory sinus arrhythmia in SIDS compared to controls (Fig. 5). However, we found increased power at low frequency in the HR spectrum (Fig. 6) and dispersion in the respiratory power spectrum (Fig. 7). Increased low frequency power was significantly different at $p<0.002$. Note, however, that the values for five infants fall well within those for controls and that only one of two recordings in infants 3 and 7 are clearly out of this range.

On the other hand, the respiratory band width discriminates SIDS infants from controls at all ages $(p<0.00001$; Fig. 7).

In one SIDS infant, an epoch of periodic breathing of greater than $256 \mathrm{sec}$ permitted similar detailed analysis so that we could compare the spectral peaks during periodic breathing to those during quiet breathing. Fig. 8 exhibits the time series of instantaneous heart rate and respiratory activity during periodic breathing. The heart rate was lowest during apnea and highest during the breathing phase. The low frequency power (in the 0.02 to 0.1 $\mathrm{Hz}$ band) was $4000 \%$ greater than the mean for this frequency band for the control group and $3000 \%$ greater than that for the SIDS group.

In summary, we found that all SIDS infants manifest increased variability of respiratory frequency and that there was a significant increase in the power of fluctuations in $\mathrm{HR}$ at a frequency identical to that observed during periodic breathing.

\section{DISCUSSION}

The increased power in the heart rate power spectrum at low frequencies $(0.02-0.10 \mathrm{~Hz})$, and increased dispersion in respiratory rate found in these eight SIDS infants supports previous suggestions that a defect in cardiorespiratory regulation characterizes the at-risk state. Previous suggestions were based on subtle pathologic evidence of hypoxia $(8,17)$, on physiologic defects in chemoreceptor control of breathing in three infants that died (13), on abnormal heart rate variability in one infant exposed to white noise (10), and on prolonged sleep apnea (16), and disruption of sleep cycles in siblings of sudden infant death syndrome victims (3). We confirm the deductions from these observations but cannot exclude the possibility that these changes are secondary to near-SIDS and/or GE reflux or to disturbed sleep. We extend these previous observations in demonstrating that the abnormality may affect both the cardiovascular and respiratory systems.

In spite of the limitations of a retrospective study, it is intriguing that the SIDS infants could be identified especially by increased variability in the respiratory power spectra. This was present independent of whether or not an infant had a nearSIDS episode. The pathophysiologic meaning of this observation might be uncovered by analysis of other respiratory variables such as inspiratory flow rate $(V T / T i)$ and the duty cycle (Ti) $\left.T_{\text {tot }}\right)$. Increased power in the HR power spectrum at low frequencies observed in SIDS infants may be linked to the marked exaggeration of power seen at this frequency during periodic breathing with a cycle duration of about $15 \mathrm{sec}$, i.e. both may reflect abnormal oscillations in heart rate determined by the same control system.

Of those with increased power at low frequency in the HR spectrum during quiet breathing, three were siblings of SIDS, two of whom had experienced a near-SIDS spell and one had choking spells with GE reflux. Previously, we have demonstrated that enhancement of activity of both the $\beta$-sympathetic and parasympathetic systems manifests itself through an increase in low frequency power $(0.02$ to $0.10 \mathrm{~Hz})$ in the heart rate power spectrum (Fig. 8). We also found in lambs and dogs that these oscillations increased $500-800 \%$ by blockade of the renin-angio- 
Table 2. SIDS infants*

\begin{tabular}{|c|c|c|c|c|c|}
\hline $\begin{array}{l}\text { Patient and age } \\
\text { (weeks) }\end{array}$ & $\begin{array}{c}\overline{\mathrm{HR}} \\
\text { (beats } / \mathrm{min} \text { ) }\end{array}$ & $\overline{R R}$ & $\begin{array}{c}\text { Band width } \\
(\mathrm{Hz})\end{array}$ & $\begin{array}{c}\text { Power } \\
\text { low frequency }\end{array}$ & $\begin{array}{c}\text { Power } \\
\text { high frequency }\end{array}$ \\
\hline 1. 8 & $121.2 \pm 2.5 \dagger$ & 25 & 0.32 & $1.87 \times 10^{-4}$ & $11.22 \times 10^{-5}$ \\
\hline 2. 18 & $129.3 \pm 1.8$ & 33 & 0.32 & $8.47 \times 10^{-5}$ & $6.56 \times 10^{-5}$ \\
\hline 3. 7 & $138.6 \pm 2.2$ & 37 & 0.50 & $1.22 \times 10^{-4}$ & $0.99 \times 10^{-5}$ \\
\hline 4. 10 & $124.3 \pm 1.7$ & 30 & 0.37 & $7.01 \times 10^{-5}$ & $4.53 \times 10^{-5}$ \\
\hline 5. 4 & $123.4 \pm 1.5$ & 44 & 0.55 & $4.71 \times 10^{-5}$ & $\ddagger$ \\
\hline 7. 3 & $109.7 \pm 2.0$ & 41 & 0.62 & $3.08 \times 10^{-4}$ & $9.70 \times 10^{-5}$ \\
\hline 8. 8 & $127.7 \pm 1.8$ & 35 & 0.35 & $8.09 \times 10^{-5}$ & $9.95 \times 10^{-5}$ \\
\hline
\end{tabular}

* The power columns indicate energy in low frequency band of heart rate power spectrum (dimensionless) and energy in high frequency band of heart rate power spectrum (dimensionless). $\overline{\mathrm{RR}}$, mean respiratory rate.

$\dagger$ Mean \pm SD

$\ddagger$ Respiratory peak extends beyond $1 \mathrm{~Hz}$, the upper limit of our spectral window.

Table 3. Control infants*

\begin{tabular}{|c|c|c|c|c|c|}
\hline $\begin{array}{c}\text { Age } \\
\text { (weeks) }\end{array}$ & $\begin{array}{c}\overline{\mathrm{HR}} \\
\text { (beats/min) }\end{array}$ & $\overline{\mathrm{RR}}$ & Band width & $\begin{array}{c}\text { Power } \\
\text { low frequency }\end{array}$ & $\begin{array}{c}\text { Power } \\
\text { high frequency }\end{array}$ \\
\hline 3 & $155.7 \pm 1.2$ & 39 & 0.17 & $3.47 \times 10^{-5}$ & $0.66 \times 10^{-5}$ \\
\hline 10 & $125.0 \pm 0.9$ & 31 & 0.18 & $3.37 \times 10^{-5}$ & $1.30 \times 10^{-5}$ \\
\hline 4 & $148.7 \pm 1.4$ & 38 & 0.22 & $5.96 \times 10^{-5}$ & $0.31 \times 10^{-5}$ \\
\hline 18 & $151.8 \pm 1.5$ & 34 & 0.20 & $3.61 \times 10^{-5}$ & $1.68 \times 10^{-5}$ \\
\hline 4 & $136.3 \pm 2.0$ & 29 & 0.25 & $2.73 \times 10^{-5}$ & $2.54 \times 10^{-5}$ \\
\hline 1.5 & $155.9 \pm 1.8$ & 29 & 0.32 & $6.22 \times 10^{-5}$ & $3.57 \times 10^{-5}$ \\
\hline 6 & $145.9 \pm 0.6$ & 44 & 0.25 & $0.80 \times 10^{-5}$ & $0.56 \times 10^{-5}$ \\
\hline 20 & $112.4 \pm 1.4$ & 25 & 0.17 & $5.62 \times 10^{-5}$ & $7.14 \times 10^{-5}$ \\
\hline 4 & $129.3 \pm 1.1$ & 33 & 0.21 & $3.07 \times 10^{-5}$ & $1.78 \times 10^{-5}$ \\
\hline 4 & $137.9 \pm 1.9$ & 37 & 0.20 & $7.73 \times 10^{-5}$ & $4.54 \times 10^{-5}$ \\
\hline 5 & $148.6 \pm 1.8$ & 38 & 0.17 & $9.05 \times 10^{-5}$ & $1.25 \times 10^{-5}$ \\
\hline 12 & $117.7 \pm 1.9$ & 24 & 0.25 & $6.06 \times 10^{-5}$ & $14.3 \times 10^{-5}$ \\
\hline 8 & $123.7 \pm 0.9$ & 28 & 0.10 & $2.36 \times 10^{-5}$ & $1.37 \times 10^{-5}$ \\
\hline 14 & $129.6 \pm 1.9$ & 36 & 0.23 & $8.23 \times 10^{-5}$ & $2.51 \times 10^{-5}$ \\
\hline 26 & $121.7 \pm 2.2$ & 33 & 0.12 & $9.51 \times 10^{-5}$ & $12.9 \times 10^{-5}$ \\
\hline
\end{tabular}

* Columns are as in Table 2.

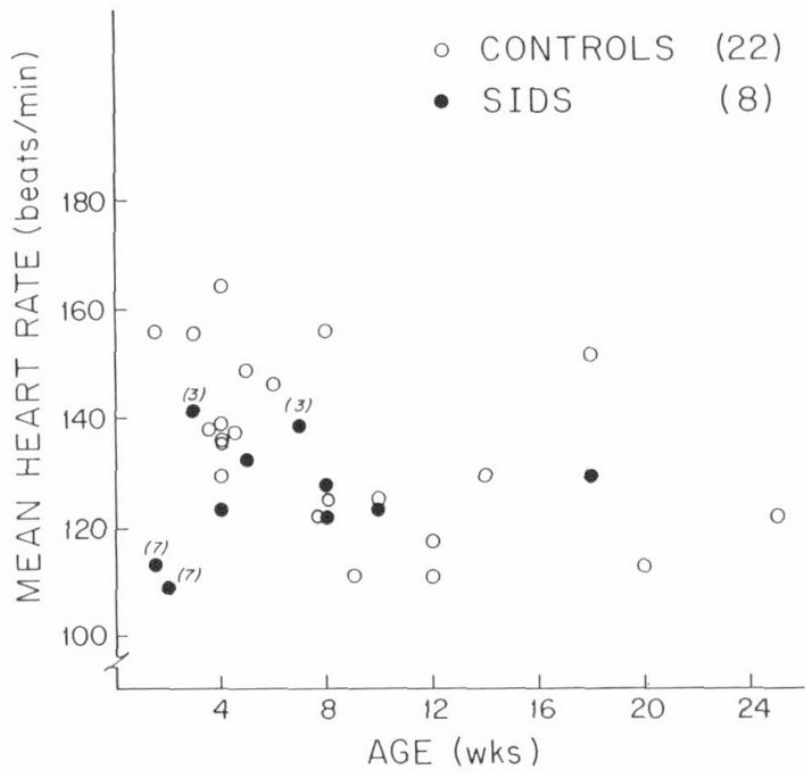

Fig. 3. Mean heart rate versus age for SIDS group (-) and control group $(\mathrm{O})$.

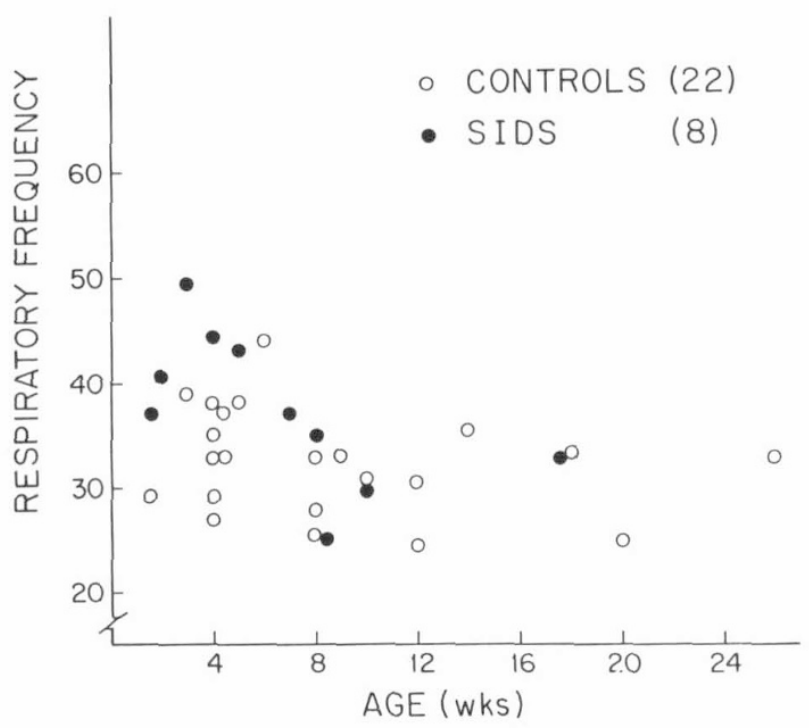

Fig. 4. Mean respiratory rate versus age for SIDS group (-) and control group $(O)$. 


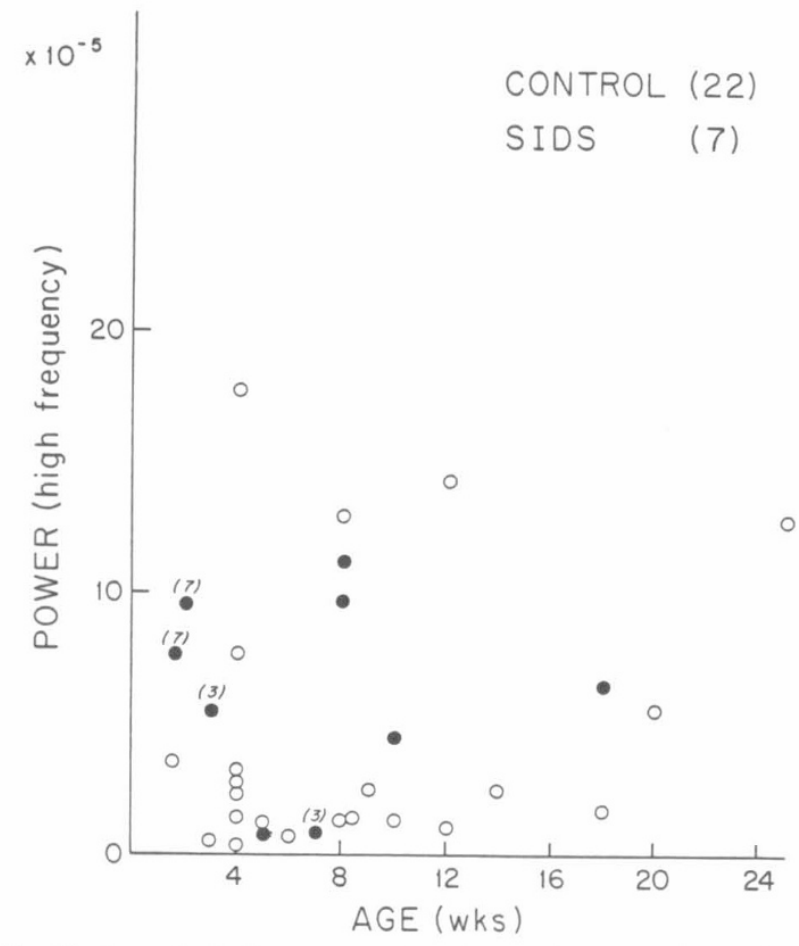

Fig. 5. Power in HR power spectrum in respiratory peak (respiratory sinus arrhythmia) versus age (SIDS, $\bullet$; controls, O).

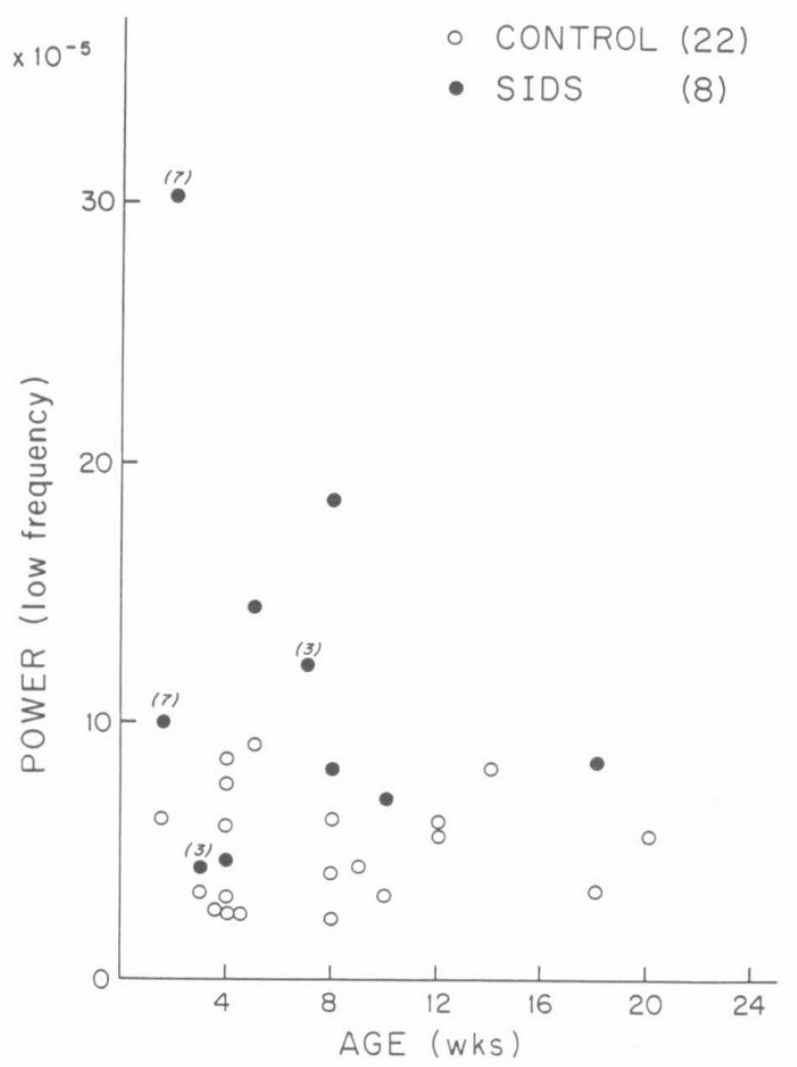

Fig. 6. Power in HR power spectrum at low frequency $(0.02-0.10$ $\mathrm{Hz}$ ) versus age. (SIDS, $\bullet$; controls, O).

tensin system with converting enzyme inhibitor (personal observations). We interpreted these results as showing that tonic activity of the renin-angiotensin system normally damps fluctuations in peripheral vasomotor tone and that blockade permits maximal fluctuations in blood pressure which are then translated into heart rate fluctuations through the mediation of the autonomic nervous system. It has been documented in a canine model that acute hypoxia (14) of even a mild degree can signifi-

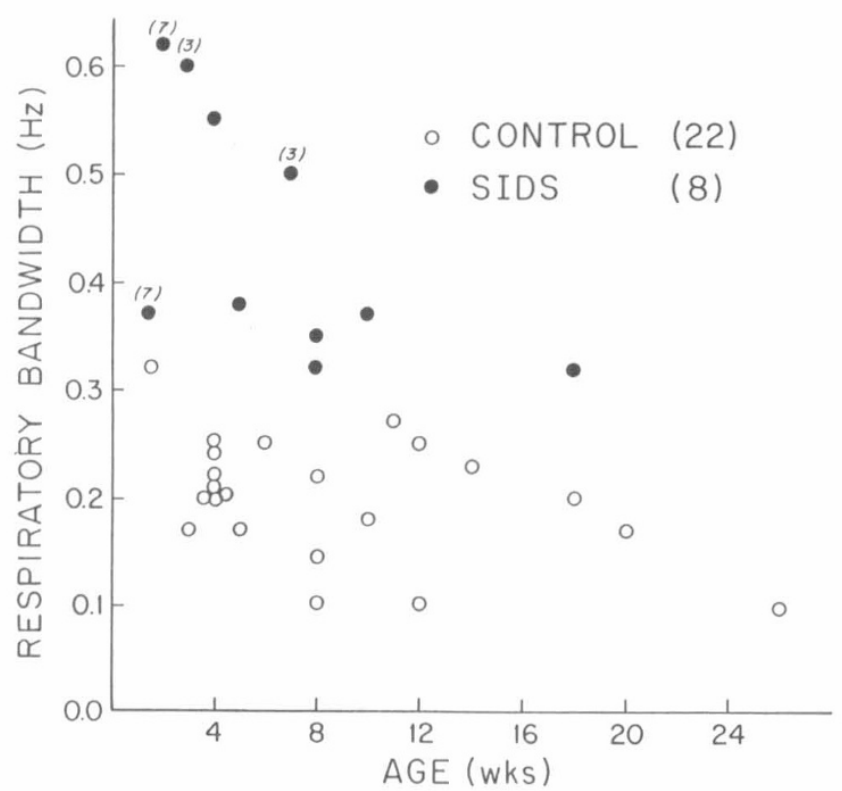

Fig. 7. Respiratory band width versus age. (SIDS, - controls, O).
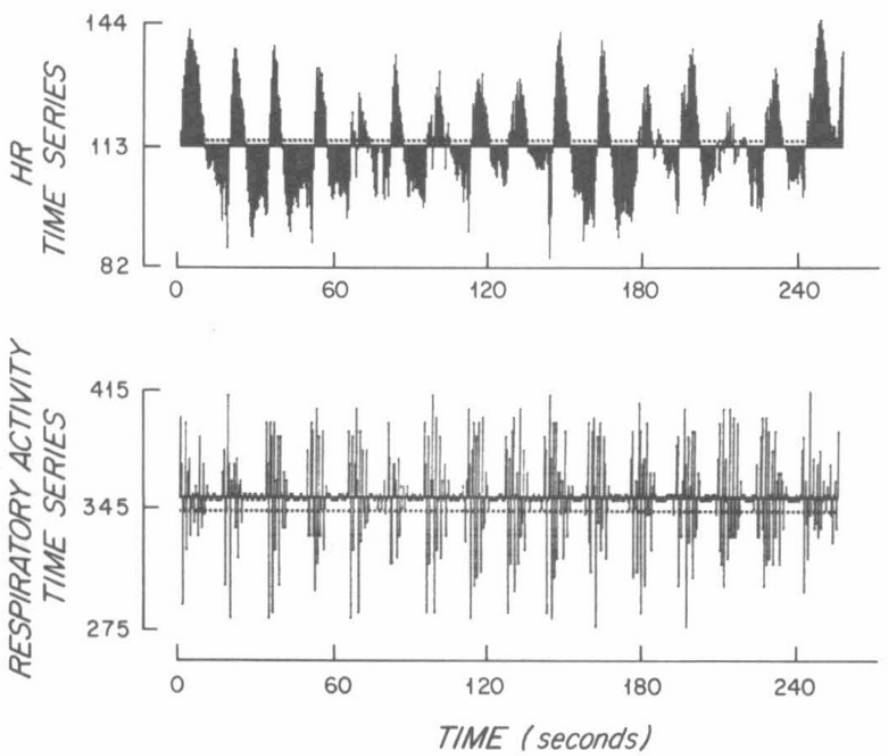

Fig. 8. Instantaneous HR (top) and respiratory activity (bottom) time series during an epoch of periodic breathing.

cantly inhibit angiotensin-converting enzyme. In preliminary experiments, we have found in chronically instrumented newborn lambs that $17 \% \mathrm{O}_{2}$ breathing enhances by approximately $90 \%$ the power in low frequency HR fluctuations. Could the mechanism of cardiorespiratory instability observed in SIDS infants manifested by frequent apneas, periodic breathing, enhanced low frequency heart rate fluctuations, lower mean heart rate, and dispersion in respiratory frequency be critically linked to the inhibition of one of the infant's major cardiovascular regulatory systems, the renin-angiotensin system? If so, it is not difficult to conceive of a self-regenerative positive feedback loop initiated by the inhibition of angiotensin-converting enzyme which may either terminate in a change in sleep state or in arousal which breaks the vicious cycle, or, if not, in death.

Finally, we must consider the possibility that the observed differences in HR and ventilation power spectra were the result of disturbed sleep. We were unable to identify differences by visual inspection of analog recordings. Nevertheless, two possibilities must be considered. First, use of an electronic monitor might alter the quality of sleep. However, this form of surveillance was not used in three infants, one of whom demonstrated the greatest power at low frequency in the HR spectrum. Second, 
parental anxiety might prompt differences in parent-infant interaction that in turn might be reflected in altered sleep or autonomic function. If so, this might explain many of the subtle changes in HR and breathing pattern observed in near-SIDS and siblings $(3,5,6)$.

We cannot determine from this retrospective study whether the abnormalities in heart rate and respiratory spectra identified in these infants are causally related to SIDS. Our results suggest the need for a prospective study in which similar data could be examined prior to the occurrence of symptoms.

Acknowledgments. We acknowledge the expert support of Dr. Adrian Wilson, Department of Medical Physics, Royal Haillamshire Hospital, Sheffield, England and Dr. Stephen Burns, Harvard-MIT Biomedical Engineering Center for their suggestions and implementation of electronic preprocessing methods to eliminate wow and flutter of the time base of the cassette record signals. We thank Robert Kenet for useful discussions and advice with regard to the digital processing algorithms.

\section{REFERENCES}

1. Akselrod S, Gordon D, Ubel FA, Shannon DC, Barger AC, Cohen RJ 1981 Power spectrum analysis of heart rate fluctuation: a quantitative probe of beat-to-beat cardiovascular control. Science 213:220

2. Guilleminault C, Ariagno RL, Forno LS, Nagel L, Baldwin R, Owen M 1979 Obstructive sleep apnea and near miss for SIDS. 1. Report of an infant with sudden death. Pediatrics 63:837
3. Harper RM, Leake B, Hoffman H, Walter DO, Hoppenbrouwers T, Hodgman J. Sterman MB 1981 Periodicity of sleep states is altered in infants at risk for the sudden infant death syndrome. Science 213:1030

4. Jacobs DLR 1974 Introduction to Control Theory. Clarendon Press, Oxford

5. Katona PG, Egbert JR 1978 Heart rate and respiratory rate differences between pre-term and full-term infants during quiet sleep: possible implications for sudden infant death syndrome. Pediatrics 62:1

6. Kelly DH, Shannon DC 1979 Periodic breathing in infants with near-miss sudden infant death syndrome. Pediatrics 63:355

7. Kitney RI, Rompelman O 1980 The Study of Heart Rate Variability. Clarendon Press, Oxford

8. Naeye RL 1973 Pulmonary arterial abnormalities in the sudden infant death syndrome. N Engl J Med 289:1167

9. Porges SW 1984 Perspectives in Cardiovascular Psychophysiology. Guilford Press, New York, in press

10. Salk L, Grellong BA, Deitrich J 1974 Sudden infant death. Normal cardiac habituation and poor autonomic control. N Engl J Med 291:219-222

11. Sayers B McA 1973 Analysis of heart rate variability. Ergonon 16:17

12. Schluter PS 1981 Design and evaluation of a bedside cardiac arrhythmia monitor. PhD thesis, Massachusetts Institute of Technology

13. Shannon DC, Kelly D 1977 Impaired regulation of alveolar ventilation and the sudden infant death syndrome. Science 197:367

14. Stalcup SA, Lipset JS, Legant PM, Leuenberger PJ, Mellins RB 1979 Inhibition of converting enzymes activity by acute hypoxia in dogs. J Appl Physiol 46:227

15. Stein IM, Shannon DC 1975 The pediatric pneumogram: a new method for detecting apnea in infants. Pediatrics 55:598

16. Steinschneider A: Prolonged apnea and the sudden infant death syndrome: clinical and laboratory observations. Pediatrics 50:646

17. Takashima S, Armstrong D, Becker L, Bryan AC 1978 Cerebral hypoperfusion in the sudden infant death syndrome? Brainstem gliosis and vasculature. Ann Neurol 4:257

\title{
Prenatal Thyroid Function Abnormalities in Infants with Idiopathic Respiratory Distress Syndrome
}

\author{
VAL ABBASSI, JAMES ADAMS, DANIEL DUVALL, AND ELIZABETH PHILLIPS \\ Department of Pediatrics, Georgetown University School of Medicine, Washington, D. C. 20007
}

\begin{abstract}
Summary
Thyroxine $\left(\mathrm{T}_{4}\right)$, triiodothyronine $\left(\mathrm{T}_{3}\right)$, and thyrotropin (TSH) concentrations were measured on cord sera of 21 preterm infants with idiopathic respiratory distress syndrome (IRDS) and were compared to the values obtained from 15 healthy preterm infants. When $\log T_{3}, \log T_{4}$, and $\log$ TSH were considered as the dependent variables in the multivariate test Hotelling-LawleyTrace, there was an overall difference between the two groups: $F$ $=3.94, p=0.03$. When $\log \mathrm{T}_{3}$ and $\log \mathrm{T}_{4}$ were considered separately in an analysis of covariance, there was a significant difference between the two groups for $\log T_{3}$ after adjusting for birth weight and gestational age $(F=7.98, p=0.008)$. However, for $\log \mathrm{T}_{4}$ and TSH, there was no difference between the IRDS and control infants. These findings exclude the possibility of antenatal thyroid dysfunction in babies with IRDS. An explanation for reduced cord blood $T_{3}$ concentration in infants with IRDS

Received November 11, 1983; accepted February 10, 1984.

Correspondence may be addressed to Val Abbassi, M.D., Department of Pediatrics, Georgetown University Hospital, 3800 Reservoir Road, N.W., Washington, D. C. 20007.

D. C.
\end{abstract}

is lacking at present. Extrathyroidal factors that predispose to IRDS may also affect peripheral $T_{3}$ generation. Alternatively, one might postulate that there is relative immaturity of both peripheral $\mathrm{T}_{3}$ generating pathway and lung development in infants who develop IRDS.

\section{Abbreviations}

\section{TSH, thyrotropin}

$T_{3}$, triiodothyronine

$\mathrm{T}_{4}$, thyroxine

IRDS, idiopathic respiratory distress syndrome

Because of the apparent role of thyroid hormones in lung maturation (7), cord blood content of iodothyronines and TSH has been extensively evaluated with interest. Several investigators have found reduced thyroid hormone concentration in cord blood of such babies $(4,5,14)$. Thus, they have suggested a thyroid role in the pathogenesis of IRDS. Such findings have been disputed by others $(9,10)$. In a previous study, we reported that cord blood $\mathrm{T}_{3}$ concentration in babies with IRDS was lower 